EPJ manuscript No.

(will be inserted by the editor)

\title{
Free Cooling Phase-Diagram of Hard-Spheres with Short- and Long-Range Interactions
}

\author{
S. Gonzalez ${ }^{1 \mathrm{a}}$, A. R. Thornton ${ }^{12}$, and S. Luding ${ }^{1 \mathrm{~b}}$ \\ 1 Multiscale Mechanics (MSM), CTW, MESA+, University of Twente, P.O. Box 217, 7500 \\ AE Enschede, The Netherlands \\ 2 Mathematics of Computational Science, Dept. of Appl. Math., University of Twente, P.O. \\ Box 217, 7500 AE Enschede, The Netherlands
}

\begin{abstract}
We study the stability, the clustering and the phase-diagram of free cooling granular gases. The systems consist of mono-disperse particles with additional non-contact (long-range) interactions, and are simulated here by the event-driven molecular dynamics algorithm with discrete (short-range shoulders or wells) potentials (in both $2 \mathrm{D}$ and 3D). Astonishingly good agreement is found with a mean field theory, where only the energy dissipation term is modified to account for both repulsive or attractive non-contact interactions. Attractive potentials enhance cooling and structure formation (clustering), whereas repulsive potentials reduce it, as intuition suggests. The system evolution is controlled by a single parameter: the non-contact potential strength scaled by the fluctuation kinetic energy (granular temperature). When this is small, as expected, the classical homogeneous cooling state is found. However, if the effective dissipation is strong enough, structure formation proceeds, before (in the repulsive case) non-contact forces get strong enough to undo the clustering (due to the ongoing dissipation of granular temperature). For both repulsive and attractive potentials, in the homogeneous regime, the cooling shows a universal behaviour when the (inverse) control parameter is used as evolution variable instead of time. The transition to a non-homogeneous regime, as predicted by stability analysis, is affected by both dissipation and potential strength. This can be cast into a phase diagram where the system changes with time, which leaves open many challenges for future research.
\end{abstract}

\section{Introduction}

Granular gases are granular materials where the duration of a collision is much shorter than the typical collision time $1 / 2 / 3456678$. This situation can be obtained by either placing a dilute particle system in a micro-gravitational environment (e.g. during a parabolic flight [9]), or experimentally easier, by feeding the system with energy such that a gaseous steady state appears (e.g. by vertically vibrating the enclosure [10 11]). For a granular gas, in the dilute limit, binary collisions dominate over multiple colli-

\footnotetext{
a e-mail: tsuresuregusa@gmail.com

b e-mail: s.luding@utwente.nl
} 
sions. Contrary to molecular gases, granular gases are dissipative. So the continuous loss of kinetic energy due to collisions not only makes the gas cool down but is also accompanied by collective phenomena such as cluster formation or shear banding. Granular gases are subject to instabilities and cluster formation [1231061314 15], deviations from the Maxwell-Boltzmann velocity distribution [16 17], phase transitions [18] and the formation of vortices [3].

The instability that leads to cluster formation is exclusively an effect of dissipation during collisions for the case of hard spheres [2, and thus should be enhanced by attractive potentials and diminished by repulsive potentials between the particles, examples of which are electrically charged or self- gravitating particles [1920. Such particle systems with attractive long-range interactions can be found, for example, in dry powders, electrostatic coating processes or in space. In the latter case, huge mass distributions of interstellar dust clouds, or dense granular rings and disks around central bodies can be affected by considerable self-gravitation 21 22 237]. Electrically charged granular media are, in nature and industrial processes, the rule rather than the exception (see Ref. 24] and references therein). However, in real systems, purely repulsive potentials are rare; in general there is an attractive well inside the repulsive barrier, which can lead to stable clustering of a granular gas 25.

This study is devoted to the behaviour of free cooling granular media as studied, e.g. by Luding and Herrmann [6], with additional non-contact repulsive or attractive potentials, as reported also in the PhD theses by S. Miller [13] and M.-K. Müller [19]. Here we focus on the dilute limit and the interactions are identical for all particles, (i.e. attractive and repulsive potentials are not active at the same time and neither are di-polar, e.g. magnetic, interactions considered [26 27]). Simulations are compared to the mean-field theory by Müller and Luding [20 28, which only features a modified cooling due to either repulsion or attraction. Our goal is to understand how dissipation and non-contact interactions act together, and what kind of dynamics is caused by the interplay of both mechanisms active at the same time in a free cooling granular system.

In section 2 we present the $2 \mathrm{D}$ hydrodynamic equations for our system. We introduce the modified cooling rate due to long-range interaction in Sec. 3. We introduce our simulation method in Sec. 4, and continue with numerical results in Sec. 5. A preliminary phase-diagram is introduced in Sec. 6 and the conclusions and perspectives of future work finalise the paper in Sec. 7.

\section{Classical Granular Gas}

In this section we review the theory of the free cooling granular gas, which is a nice reference case since it is analytically solvable under a few simplifying assumptions, as e.g. homogeneity that leads to the homogeneous cooling state (HCS), and is known in the literature as Haff's law [1].

\subsection{Homogeneous Free Cooling Theory}

A free cooling granular gas in its HCS [1] dissipates kinetic energy $K$, as governed by the equation

$$
K(\tau) / K(0)=(1+\tau)^{-2}
$$


with the rescaled time $\tau=\left(1-r^{2}\right) t /\left[2 \mathfrak{D} t_{E}^{0}\right]$. $\mathfrak{D}$ is the dimension of the system, $r$ the coefficient of restitution, and $t_{E}^{0}$ the initial Enskog collision rate [15]29],

$$
t_{E}=\frac{d \sqrt{\pi}}{2^{\mathfrak{D} \mathfrak{D} \nu g_{\mathfrak{D}}}(\nu) \sqrt{T_{g} / m}},
$$

with the (time-dependent) granular temperature $T_{g}=\frac{2}{2} \frac{K(t)}{N}$, i.e., twice the kinetic energy per particle per degree of freedom, while $T=(\mathfrak{D} / 2) T_{g} / m$ is the square of the velocity fluctuations. The diameter of the particle is $d, \nu$ is the packing fraction of the system and $m$ the mass of the particles. In 2D, the pair correlation function at contact $g_{\mathfrak{D}}$ is given, approximately for low to moderate densities, by [30]31]

$$
g_{2}(\nu)=\frac{1-7 \nu / 16}{(1-\nu)^{2}},
$$

and in $3 \mathrm{D}$ by

$$
g_{3}(\nu)=\frac{1-\nu / 2}{(1-\nu)^{3}} .
$$

Improved formulae for higher density can be found in 29|32]33|34.

\subsection{Hydrodynamic Equations}

The hydrodynamic equations for a granular gas are explained in detail in [34. For the sake of brevity, we present a summarised version.

The continuity equation for the mass density, $\rho$, reads:

$$
\frac{D \rho}{D t}+\rho \frac{\partial u_{i}}{\partial x_{i}}=0
$$

where $u_{i}$ is the velocity in the $x_{i}$ direction. Momentum conservation in absence of gravity gives:

$$
\rho \frac{D u_{i}}{D t}=-\frac{\partial \sigma_{i j}}{\partial x_{j}},
$$

where $\sigma_{i j}$ is the stress tensor. The energy balance reads:

$$
\rho \frac{D}{D t} T=-\sigma_{i k} \frac{\partial u_{i}}{\partial x_{k}}-\frac{\partial q_{k}}{\partial x_{k}}-I,
$$

where $q_{k}$ is the heat flux and $-I=-I_{0}=-\hat{\gamma} T$ the energy density dissipation rate in the absence of any additional forces, i.e. with potential energy $\phi=0$. For the explicit formulation of the coefficients see [13]15]29]34.

The HCS for a freely cooling gas is found by taking all the spatial derivatives in the hydrodynamic equations equal to zero, leaving just one equation for the temperature. The remaining fields - density and velocity in each component - remaining homogeneous and constant: $\nu=\nu_{0}, \mathbf{u}=0$. Knowing that $\hat{\gamma} \propto \sqrt{T}$, and rescaling time, the result is directly Eq. (1). This solution to the simplified system of equations is what Haff derived from simple mechanical arguments in his seminal paper 1]. 


\subsection{Cluster Instability}

The homogeneous cooling state, however, is not always a stable solution for the system. For the hard-sphere potential, when the system size is large enough (at a given dissipation), the homogeneous cooling becomes unstable and shear and clustering modes appear in the system. For the attractive potential we expect that the cluster instability will be always enhanced, and on the contrary, for the repulsive cased reduced.

The spontaneous formation of clusters in a force-free cooling granular gas can be understood by simple arguments [2 35]: consider density fluctuations in an otherwise homogeneous granular gas. In denser regions the particles collide more frequently than in more dilute regions, therefore, dense regions cool faster than dilute regions and thus the local pressure decays faster as well. The resulting pressure gradient causes a flux of particles into these regions of higher density, which leads to further increase of the density. Hence, small fluctuations of the density are enhanced, which leads to the formation of clusters.

Simulation and hydrodynamic equations are both non-dimensionalised, with the particle radius and the initial granular temperature. The potential strength is scaled by the initial too temperature and hence is dimensionless. Fig. 1 shows snapshots for the same initial conditions after $7 \times 10^{5}$ collisions for an event-driven simulation with non-contact interactions, where all details are given in the following sections. We use relatively weak potentials where the ratio of the potential at contact to the initial granular temperature is $-10^{-5}, 0,10^{-5}$, for attractive, neutral, and repulsive potentials, respectively. The three systems present similar clusters in shape but their evolutions and structures are different; in particular, clusters for the attractive case are denser than in the hard-sphere case, while for the repulsive case they are relatively more dilute. The following section introduces the theoretical mean field approach we will use to study the homogeneous cooling regime that occurs before the clustering.
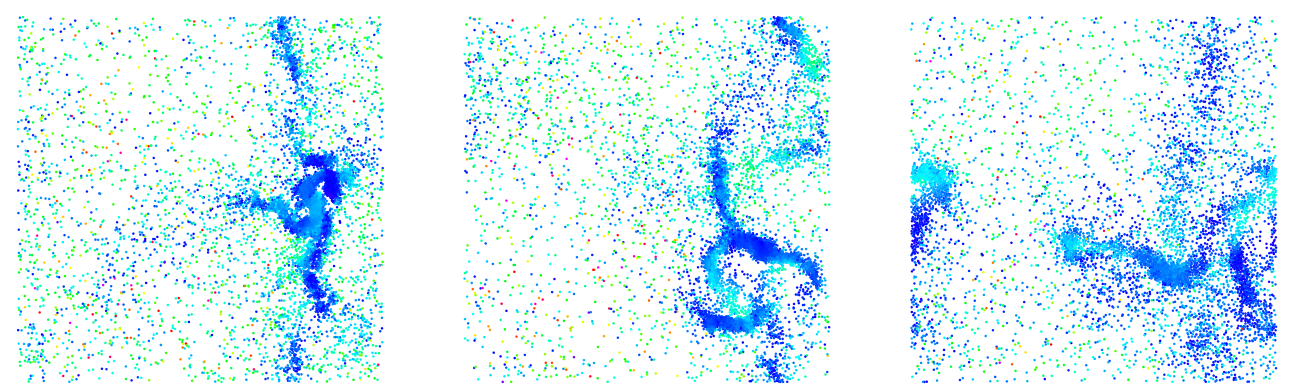

Fig. 1. Snapshots after $7 \times 10^{5}$ collisions for systems with weakly attractive, neutral, and weakly repulsive potentials, from left to right. Number of particles $N=6400$, density $\nu=$ 0.0578 and dissipation $r=0.65$. The colour code indicates the kinetic energy per particle, from blue (slow), to yellow (average) and red (fast).

\section{Dissipation rate modification due to attraction and repulsion}

Haff's law is valid only for particles with hard-core interaction in the homogeneous cooling state (HCS). For long range interaction, Müller and Luding [20 28] predicted, using a modified pseudo-Liouville operator formalism, a reduced cooling rate due to 
the repulsive forces and an increased rate due to attractive forces (extending the results of [36]). In their theory, the ratio of the potential at contact, $\phi$ (see subsection 4.1), to the temperature is the control parameter,

$$
\Gamma=\frac{|\phi|}{T_{g}}
$$

with different sign convection as in Ref. [20], where $\Gamma^{*}=\phi / T_{g}$ was used in some equations. Since the theory is a simple mean field theory, the shape of the potential does not enter in the formulation, only its value at contact [20].

Since the cooling rate is modified, the transport coefficients of the system will be modified accordingly. We will focus only on the change produced in the dissipation rate, since it is the dominant term controlling the dynamics of the system. Namely, the modified dissipation rate is:

$$
I=I_{0} \psi(\Gamma):=I_{0} \begin{cases}1 & : \text { Haff } \\ \exp (-\Gamma) & : \text { Repulsive } \\ (2-\exp (-\Gamma)) & : \text { Attractive }\end{cases}
$$

These modifications were derived for $3 \mathrm{D}$ systems by computing the average effect of a long-range potential in the collision frequency of a granular system with a pseudoLiouville operator approach. We will use them directly in $2 \mathrm{D}$ accounting for the different dimensionality in the pair correlation function at contact and the numerical factors in the Enskog collision time. This is justified - and confirmed by our simulations - since the integration of the Liouville operator considers collisions in a plane due to angular momentum conservation, and hence only the prefactor is different between $3 \mathrm{D}$ and 2D, while the functional form in $\Gamma$ remains identical.
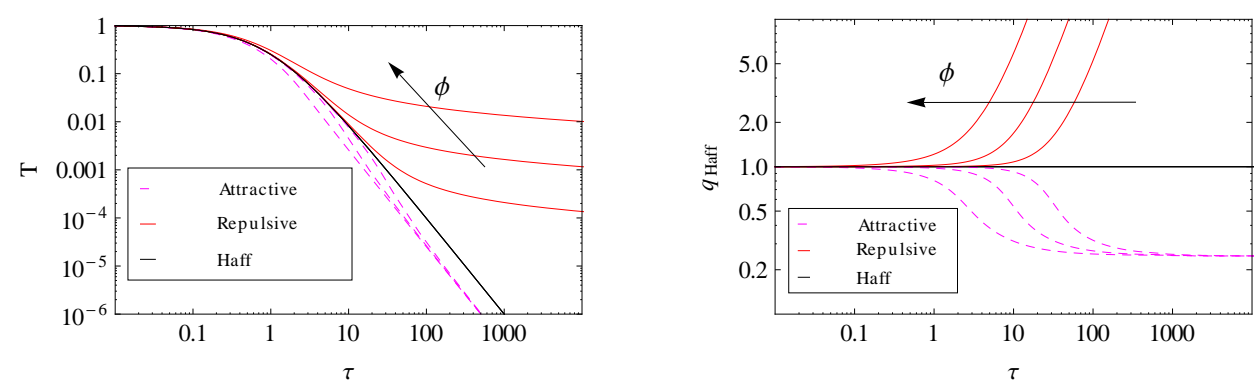

Fig. 2. Cooling of granular systems. Left: temperature evolution for hard-spheres (Haff's law, solid black), repulsive interaction and attractive interaction as given by the numerical solution of the temperature equation (5) considering the dissipation term as in Eq. (7) for different values of $\phi=10^{-3}, 10^{-2}, 10^{-1}$. Right: quality factor $q_{\text {Haff }}=T / T_{\text {Haff }}$ for the same long range potentials as on the left plot.

The homogeneous cooling with long range interaction is given by Eq. (5) taking all the spatial derivatives equal to zero and using the modified cooling rate from eq. 7. This was numerically solved (we used Mathematica 8) and Fig. 2 shows the evolution of the temperature for three cases: attractive $(\phi<0)$, repulsive $(\phi>0)$ and no long-range interaction (Haff's law, $\phi=0$ ).

Physically, Fig. 2 says the following: In Haff's case, the dynamics of the systems becomes slower as time advances, making the dissipation slower, and so on, as long 
as the system is homogeneous [1]. In the presence of non-contact forces, the system can have at least two different regimes: at the beginning, the thermal energy is larger than the repulsive/attractive energy, its effect being negligible. As the system cools down, the repulsive/attractive barrier will start to be felt, and the cooling will be consequently modified. For the attractive force the prediction says that once the particles start to feel the attraction they will dissipate energy faster but nevertheless will retain the power law of the dissipation and keep the homogeneity.

\section{Event-Driven simulations}

Event-driven simulations have been widely used to study granular gases 3 6 13 14 15 29 35, and have shown to capture the correct behaviour when compared to kinetic theory. In what follows we present the details of the simulation algorithm for discrete potential simulations where the range of attraction is finite.

The simulations used to prove the theoretical predictions in Ref. 20] were done with continuous potentials, which make them computationally expensive and time consuming. However, the theoretical description does not consider the shape of the potential but only its repulsive barrier. Thus we complement the original theoretical and numerical work by considering discrete potentials, as readily simulated by event driven algorithms [13] that are typically much faster but - to the knowledge of the authors - could not yet be parallelised as efficiently as continuous potential simulations [13.

\subsection{Discontinuous potentials}

Discrete potentials, such as the hard sphere model, have an important advantage over more complex "soft" potentials. Between collisions the spheres or molecules experience no forces and travel on ballistic trajectories. The dynamics can be solved analytically, and the integration of the equations of motion is processed as a sequence of events rather than by fixed, small time-steps. Current event driven molecular dynamics algorithms are quite advanced and allow the simulation of large systems for the long times required to extract accurate transport properties and study, e.g., the evolution of clusters 34.37] over many orders of magnitude.
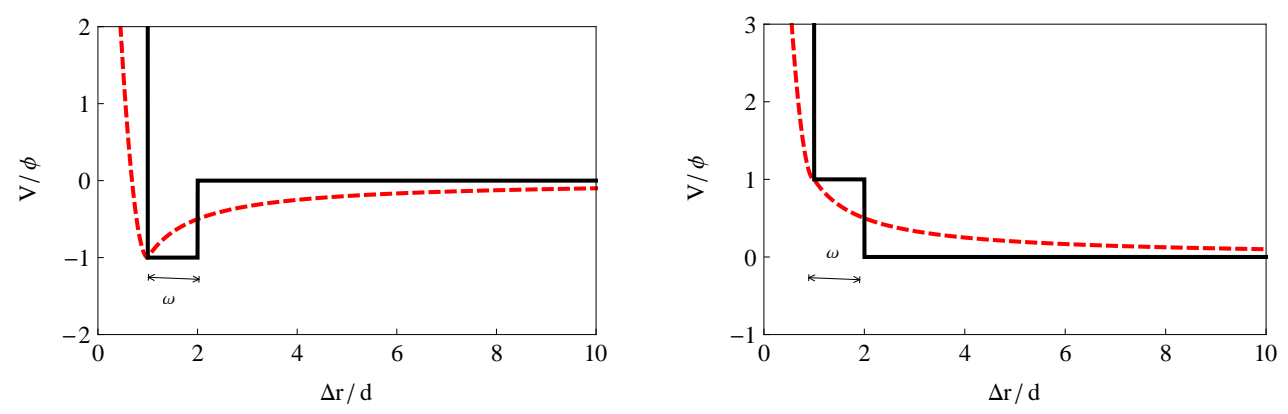

Fig. 3. Plot of the potential energy for both continuous and discontinuous models with attractive (left) and repulsive (right) potentials as a function of the inter-particle distance $r$.

For our simulations we use a two-level potential, see Fig. 3. The core is a hard sphere and there is a well (or barrier) at a distance $\omega=1.5$ (for all simulations 
unless otherwise noted) and of amplitude $\phi$. At collisions of the hard sphere cores, the relative velocity is updated only in the normal direction according to

$$
\mathbf{v}_{i j}^{\prime}=\mathbf{v}_{i j}-(1+r)\left(\mathbf{v}_{i j} \cdot \hat{\mathbf{n}}_{i j}\right) \hat{\mathbf{n}}_{i j},
$$

with normal unit vector $\hat{\mathbf{n}}_{i j}=\mathbf{r}_{i j} /\left|\mathbf{r}_{i j}\right|$, where $\mathbf{r}_{i j}$ is the distance between the particles $i$ and $j, \mathbf{v}_{i j}$ is their pre-collisional relative velocity, and the prime indicates a postcollisional quantity. At crossing the well (barrier or sink) the particles lose or gain energy instantaneously, depending on the direction of their relative motion, for details see Ref. [13. The normal velocity after crossing is:

$$
v_{n}^{*}=\sqrt{v_{n}^{2} \pm 2 \phi / m},
$$

with the normal velocity before, $v_{n}=\mathbf{v}_{i j} \cdot \hat{\mathbf{n}}_{i j}$, and where the sign depends on whether the potential is attractive or repulsive, whether the collision is outgoing or incoming, and if $v_{n}$ is large enough to cross the well or whether the particles are reflected similar to the hard core collision. For example, in the case of a rapidly incoming collision, in the attractive (repulsive) case, the particles gain (lose) energy, while for an outgoing collision this is reversed. How the sign and magnitude of this potential affect the macroscopic evolution of a free-cooling gas is the subject of the rest of the study.

\subsection{System and Preparation}

The simulation consists of a system of $N$ particles in a square in 2D (or cube, in $3 \mathrm{D})$ of side length $L$ with periodic boundary conditions. Particles are mono-disperse with diameter $d$ and mass $m$. The packing fraction of the system is given by $\nu=$ $N \pi d^{3} /\left(6 L^{3}\right)$ in $3 \mathrm{D}$ and by $\nu=N \pi d^{2} /\left(4 L^{2}\right)$ in $2 \mathrm{D}$. The simulations were carried out in DynamO, a free and open-source event-driven code [38.

\subsubsection{Initial Conditions}

The initial state is prepared as follows: (1) Start with a square lattice (2D) or a hexagonal close packing (3D), uniform random velocities and a given $\phi$. (2) Let the system equilibrate, while each particle collides at least 100 times elastically, until a homogeneous regime is reached. This is tested by looking at the density and the distribution of velocities and confirming that they are homogeneous and very close to Maxwellian, respectively. (3) Once thermalised, the velocities are scaled so $T_{0}=1$ and dissipation is turned on. From here, the system is allowed to freely cool down with a fixed particle coefficient of restitution, $r$.

\subsubsection{Simulation Units}

The simulations are performed using a non-dimensionalised system. The units of length, mass and time are set such that, $d=1, m=1$ and $T_{0}=1$. Since we are interested in the perturbative case, the potential, $\phi$, is mostly varied in the range from $10^{-5}$ to $10^{-3}$ for both, attractive and repulsive cases.

\section{Numerical Results}

In this section we present first the results for the attractive regime, followed by the repulsive case results. 


\subsection{Attractive forces}

In this subsection we analyse data for cooling with attractive potentials of different intensity and for different coefficients of restitution. The objective is to understand how the dynamics is influenced by these two factors and how they interact. We focus on the evolution of three aspects: the temperature, i.e. the degree of cooling; the velocity distribution of the particles; and, the cluster structure and size distribution.

The initial state for different systems is always the same: the distribution of particles is homogeneous, with a Maxwellian velocity distribution, and, hence, the cooling is well described by Haff's law. As time passes dissipation and the attractive potential will have time to act and modify this picture giving rise to a modified cooling dynamics and clusters.

\subsubsection{Cooling}

The theoretical prediction from Müller and Luding 20 for the cooling rate is a homogeneous state with a twice as large dissipation rate. From Eq. (7), we have for $t \rightarrow \infty$ the temperature $T_{g} \rightarrow 0$ and thus the control parameter $\Gamma \rightarrow \infty$ so that $I=2 I_{0}$. Furthermore, the theory predicts that systems with different $|\phi|$ will deviate from Haff's law at different times; the smaller $|\phi|$ the more time it takes to deviate, however, the functional form remains the same 1 .

Figure 4 shows the temperature (normalised by Haff's law) for systems with $\phi=10^{-3}, 2 \times 10^{-3}, 5 \times 10^{-3}, 8 \times 10^{-3}, 10^{-4}$ and low dissipation so the cooling is homogeneous. The cooling is well predicted by the theory only for the initial deviation from Haff's law but there is no agreement after that; simulations dissipate more energy than predicted, since the theory cannot account for the inhomogeneities in the simulations. Interestingly, the simulations do seem to follow power laws of similar slope but with different scaling factors. This can be seen around $\tau \simeq 100$ where all the curves saturate close to $q_{\mathrm{Haff}} \simeq 0.1$. Due to the long time it takes to simulate the clusterised state, we do not have data in the very long time regime.

Snapshots of the evolution for one of these low-dissipation systems can be seen in Fig. 6. Once the attractive force is larger than the thermal fluctuations, the system develops clusters. The structure of these clusters is typical for a cluster-cluster aggregation process, with an exponential decay on the cluster size (see below).

\subsubsection{Velocity Distribution}

The temperature alone is not a good indicator of the structure formation process since it also depends on $\phi$. A better indicator is to look for the deviation of the velocity distribution from a Maxwellian. For this, we focus on the evolution of the kurtosis $\beta_{2}=\mu_{4} / \mu_{2}^{2}$, where $\mu_{i}$ denotes the $i$ th central moment (and $\mu_{2}$ in particular the variance) as a function of $\Gamma$. When the $\beta_{2} \simeq 3.108$ the system is homogeneous. (It must be noted that since the system has a finite number of particles, the kurtosis does not reach the theoretical value but fluctuates near to it.) As soon as clusters appear in the system, the velocities of the particles in the cluster are more correlated and hence the distribution of velocities deviates from the Maxwellian towards higher values of $\beta_{2}$.

By studying the evolution of the system as a function of the relevant control parameter, $\Gamma$ (i.e. the strength of the non-contact potential relative to the granular

\footnotetext{
${ }^{1}$ By functional form we mean that changing the potential is equivalent to make a change of variable in time.
} 


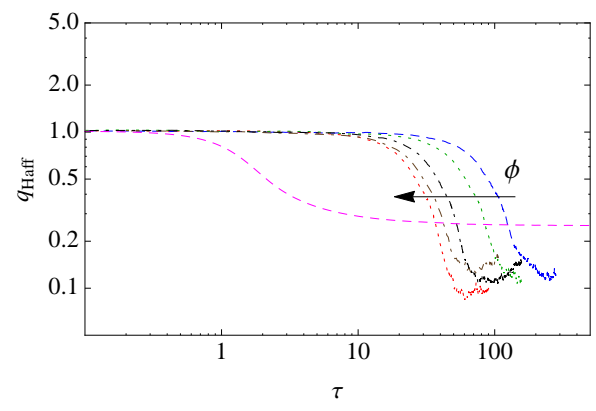

Fig. 4. Temperature evolution normalised by Haff's law with attractive potential for $r=$ 0.99, $\phi=10^{-3}, 2 \times 10^{-3}, 5 \times 10^{-3}, 8 \times 10^{-3}, 10^{-4}$ together with Haff's law (solid-black) and the theoretical prediction for $\phi=1$ (dashed magenta). The onset of cooling in the simulations is well predicted but the later cooling rate is overestimated, the magenta line runs noticeably on top of the simulations.

temperature, see Eq. 7), one is looking at the same time at the temporal evolution of the system, since $T_{g}$ is decreasing with time. The advantage of looking at $\Gamma$ instead of just at the true time of the system is that different coefficients of restitution can be compared in one plot without having to scale the temporal axis, see Fig. 2 and Eq. (4.2) in Ref. [20].

Figure 5 shows the kurtosis during the cooling for three systems with attractive potentials for different coefficients of restitution and potential strength, at a fixed density. If the cooling is homogeneous (solid and dashed lines), the deviation from the homogeneous state is due solely to the attractive potential and sets up when $\Gamma^{-1}$ is smaller than one. When the cooling is not homogeneous, i.e. inelastic clusters appear, the system deviates from the Maxwellian distribution of velocities before the attractive potential has time to act. This indicates that there are two mechanisms of clustering present in the system: inelastic cluster formation and fractal-like aggregation. Depending on $\Gamma$ one or the other will dominate.

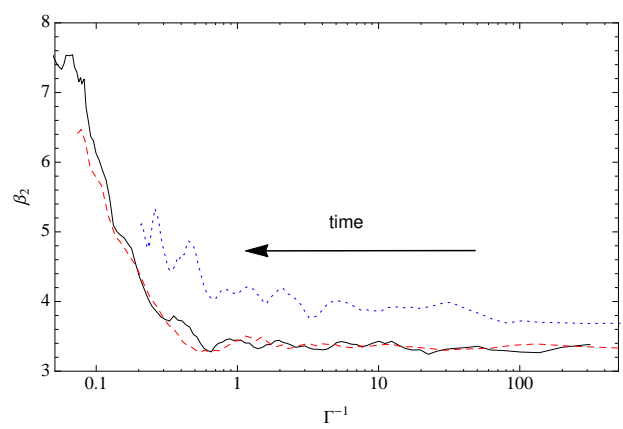

Fig. 5. Kurtosis of the velocity distribution as a function of $\Gamma$ for systems with attractive potential. Different $\phi$ collapse on the same curve as long as the coefficient of restitution is large enough. When the coefficient of restitution goes below the critical value given by HCS, the system develops dissipative clusters before the attractive cooling sets in. The bottom lines correspond to an evolution as in Fig. 6] while the upper line corresponds to an evolution as observed in Fig. 7 


\subsubsection{The cluster structure as a function of $\phi$}

Roughly, we divide the phenomenology in two different regimes quantified by $\Gamma$ : comparable $(\Gamma \sim 1)$ and small $(\Gamma \ll 1)$ short-range effects. In the first regime, the attractive potential is stronger than the kinetic energy and hence when particles collide they tend to remain bound together creating clusters in a homogeneous way; this is the fractal-like process we discussed before. The second regime is the one where the attractive potential is weak enough to let the cluster instability appear in the system; this is the inelastic cluster regime. However, once a cluster occurs, the attractive force binds it together and makes the dissipation rate to be stronger than in an equivalent cluster of hard spheres. The regime $\Gamma \gg 1$ is not considered, since it resembles the limit of cluster-cluster aggregation process [39] and the dissipation has no role to play there.

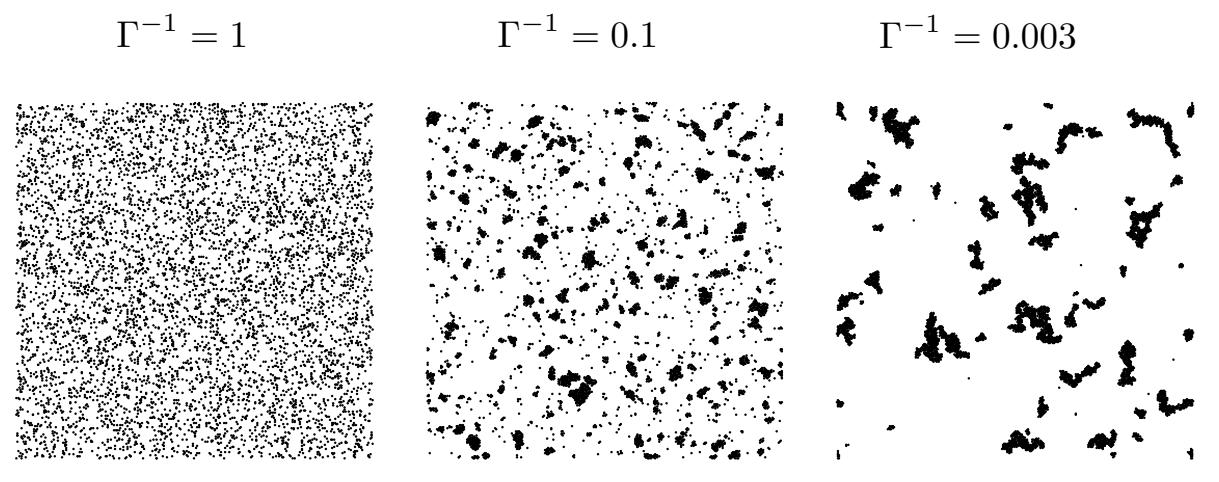

Fig. 6. Simulation snapshots at different $\Gamma$ (time increases from left to right) for a considerable attractive potential $(\phi=-1)$ for $N=6400, \nu=0.0578$ and $r=0.99$. This corresponds to a system equivalent to the lower dashed line in Fig. 5 but with a larger attractive force.

Figures 6 and 7 show snapshots of systems for decreasing $\Gamma$ from left to right, and a different coefficient of restitution and $\phi$ in each figure. In the first case, Fig. 6, with a relatively strong potential and low dissipation, the cooling is homogeneous until $\Gamma \simeq 1$ when clusters start to appear homogeneously, in a way similar to the clustercluster aggregation process. Fig. 7 shows snapshots for a system with an increased dissipation, $r=0.6$ and reduced attractive potential, $\phi=-10^{-4}$. This gives enough time for dissipation-induced clusters to appear. Once a cluster appears, since the relative velocity of its particles is smaller than the thermal fluctuations, the attractive force come into play, further increasing the dissipation inside the cluster. The effect of this is that there is no energy left to break the cluster, and once it forms, it will remain in the system with roughly the same shape, thus creating a non-homogeneous clusterisation. This can be seen from the cluster size distribution and its temporal evolution as well as from the snapshots.

Figure 8 shows the cumulative distribution function (CDF) for the evolution of the cluster size distribution for two systems, one with homogeneous clusterisation and the other with inelastic cluster formation. In the homogeneous case (left) the distribution of cluster sizes is broader than in the clusterised case (right) and there are almost an order of magnitude fewer clusters of size one for a similar maximum cluster size. 


$$
\Gamma^{-1}=3600
$$

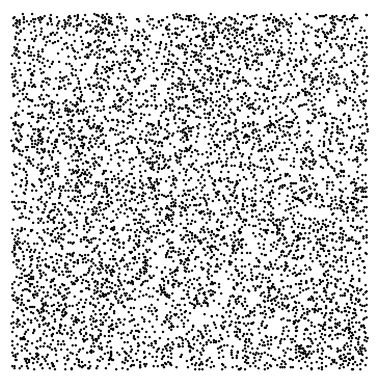

$\Gamma^{-1}=7.51$

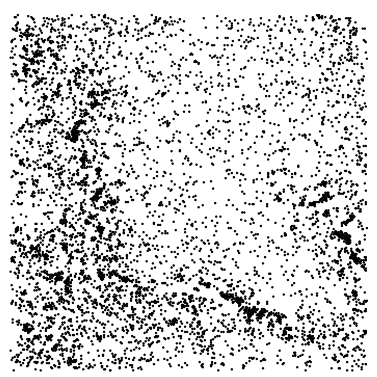

$$
\Gamma^{-1}=4.7
$$

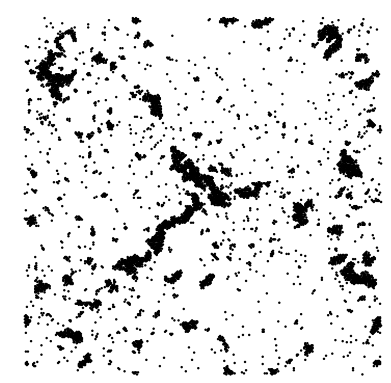

Fig. 7. Simulation snapshots at different $\Gamma$ (time increases from left to right) for a weak attractive potential $\left(\phi=-10^{-4}\right)$ for $N=6400, \nu=0.0578$ and $r=0.6$. This correspond to an evolution as the one depicted in Fig. 5. upper dotted line.

On the other hand, for the inelastic cluster formation (right), the distribution of sizes is more heterogeneous: there are plenty of clusters of small size and a few very large clusters. This can be seen also in the normalised plot, Fig. 9, where for the homogeneous case the size distribution approaches a straight line, i.e. a logarithmic function. This means that in the limit of infinite particles, there are particles of all the sizes but the probability of finding a cluster of size $n$ scales as $1 / n$. In the case of inelastic clusters, the evolution of the size distribution does not follow a clear trend.
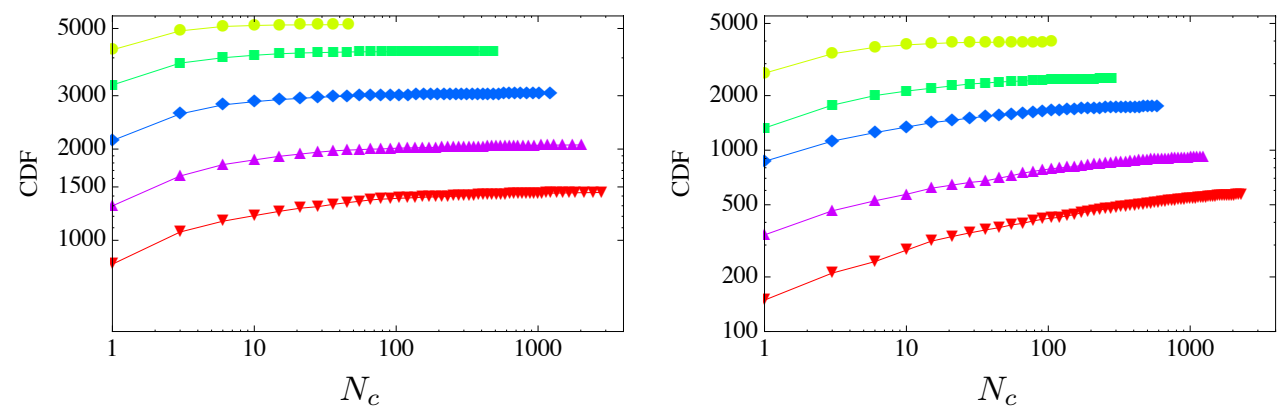

Fig. 8. Cumulative cluster size evolution as a function of cluster number $N_{c}$ (number of particles in a given cluster [6]) for a homogeneous clustering system (left) and a system with inelastic cluster formation (right). In both plots different symbols represent different stages in the evolution from more homogeneous (upper lines) to clusterised systems (bottom line). 

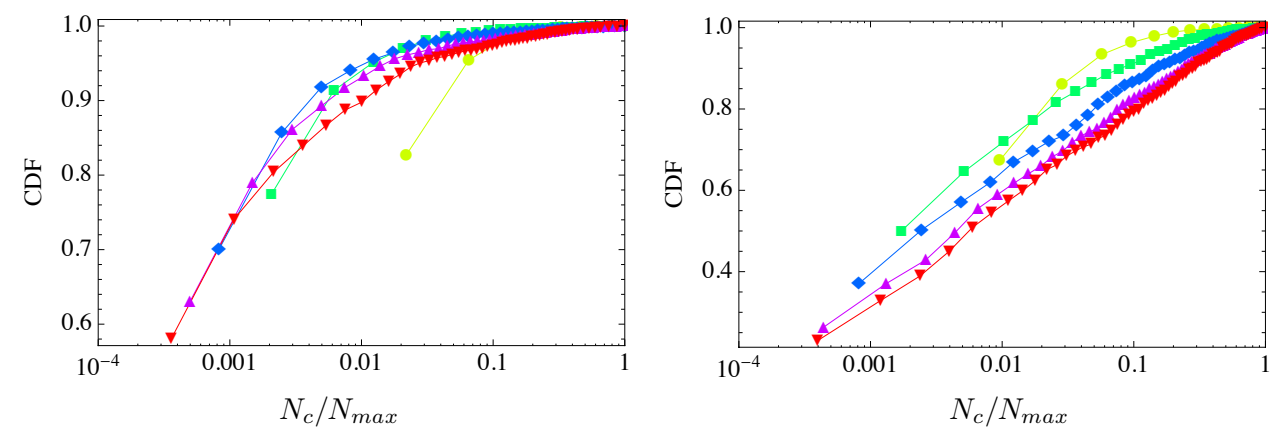

Fig. 9. Cumulative cluster size evolution normalised by the largest cluster size, $N_{\max }$. Same data as in Fig. 8 For the homogeneous case the evolution of the size distribution seems to converge to a straight line.

\subsubsection{Attractive cooling systems are always unstable}

Hard-spheres with attractive long-range interactions present condensation [40. Indeed, for the elastic case, there is a region of temperatures where the liquid and gas phases can coexist. Consequently, for a homogeneous gas of cooling hard-spheres with long-range attraction, the temperature eventually reaches the critical point where the liquid phase becomes stable, and then clusters develop. These clusters will grow due to the inelasticity of the particles to eventually reach a single cluster composed of all the particles of the system. This is independent of the density and size of the system. In this way, the free cooling of hard-spheres with attractive wells is always unstable to cluster formation disregarding the size of the system. However, the clusterisation process can be, in turn, homogeneous or inhomogeneous. It must be noted that the term homogeneous is scale-dependent: once the energy of the system is low enough particles merge into pairs breaking the local homogeneity, but remaining homogeneous in larger scale that comprises several small clusters.

This makes a fundamental difference with the free cooling of hard-spheres, where the clustering instability depends on the wavelength of the perturbation; if the system is small enough, the cooling will be always stable. We proved this by realising small $(N \approx 100)$ simulations of attractive elastic particles - the phase separation always appeared independent of the system size. Furthermore, even for two particles, if the temperature is low enough, they will eventually find each other and merge into a cluster. Thus, since the phase separation does not depend on the system size, it always appears in a free cooling system when the temperature gets low enough $(\Gamma \approx 1)$.

\subsubsection{Comparison with wet granulates}

Our model can be compared to the cooling of wet granulates, see Refs. [4142], where the authors studied cooling by using a very simple model for the interaction of two wet grains, which only accounts for the essential features of a capillary bridge: hysteresis and dissipation with a well-defined energy loss. Cooling is controlled by the probability for a bridge to break and hence logarithmically slow in the long time limit, when a percolating structure has been formed. In contrast to theirs, our model has the dissipation occurring at collision and conserves energy at the crossing of the 
energy barrier. These two microscopic differences may have a great influence on the macroscopic behaviour of the system. As we have seen, our model develops inelastic clusters, contrary to Ulrich's 42, which is homogeneous. This could be due to the strong attraction they use as initial condition $(\Gamma=1)$. A detailed comparison of the two models is beyond the scope of this study; however, it remains as an interesting open question to investigate in the future.

\subsubsection{Effects of higher density}

For the continuous potential of Ref. [20], the cooling of denser systems was shown to be not predicted by the dilute limit theory. This was due to the multi-particle interactions that occur in dense systems with long-range coupling. To see if the discrete potential reproduces this behaviour, we realised simulations analogous to the ones in Müller and Luding's paper [20].

One of the most notable features of the continuous potential simulations is that the cooling is not monotonically decreasing: the system phase-separates and the geometrical rearrangements produce a temporary increase in the kinetic energy of the system. We did not observe this increase in the temperature for the simulations with a well width of $\omega=1.5$ despite seeing the phase separation. Thus we decided to vary the potential width to see if this long-scale rearrangements are recovered with discontinuous potentials. Figure 10 shows the energy for dense systems with different well widths $\omega$ in 3D. For this density, $\nu=0.157$, all the systems present a phase transition below a critical temperature: the homogeneous system becomes unstable and the system phase-separates in a liquid and a gaseous (almost vacuum) region. This is independent of the well width $\omega$. However, as the well becomes wider, the phase separation changes qualitatively, from a percolating system with big bubbles to a system made of one big drop. For different $\phi$ the qualitative evolution is similar, only shifted to the corresponding temperature (data not shown). The discrete system also presents a peak in the kinetic energy if $\omega$ is large enough. In this case, the qualitative change from a cooling that is strictly monotonous to one that presents a peak, occurs around $\omega_{p} \approx 2.25$. It must be noted that since the strength of the potential was kept constant, the "bump" in the temperature shifts to earlier times since the potential energy of the system increases too when increasing $\omega$.

In systems where the density is so large that the particles' interactions are not binary anymore, the theory can not predict the cooling behaviour. However, some complex physics - as the increase in temperature due to geometrical rearrangements and structure formation [19] - is recovered even with a discrete potential.

\subsection{Repulsive forces}

Intuition tells us that in the repulsive case there are two regimes depending on whether the original hard-sphere system presents a homogeneous or non-homogeneous cooling. For the homogeneous cooling it is obvious that the repulsive potential will not enhance the clustering and the cooling will remain homogeneous. In the non-homogeneous case, the repulsive forces will act against the cluster formation since they tend to separate particles. However, one can expect that if the potential is weak enough, it will not affect the formation of clusters, at least temporarily. Eventually, the temperature drops under the repulsive energy and the clusters are eliminated. Quantifying this statement is the subject of the following subsections. 


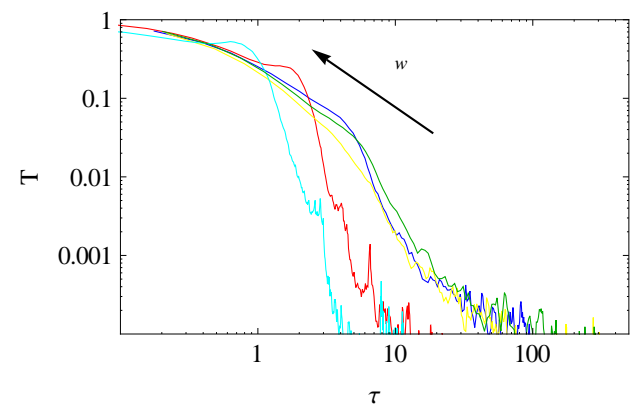

Fig. 10. Cooling for dense $(\nu=0.157)$ systems with large dissipation, $r=0.85$ and different potential width $w=1.1,1.5,2,2.5,3$ but fixed $\phi=0.1$. As the well becomes wider, the kinetic energy presents a peak due to large-scale reorganisation.

\subsubsection{Homogeneous Cooling}

Contrary to the attractive case, the mean field theory for repulsion is in great agreement with the simulations. The density remains homogeneous and the temperature follows Eq. (5). This is a strong result as there are no free parameters in the theory, everything is determined by the potential at contact. Fig. 11 shows the temperature for two systems, in both $3 \mathrm{D}$ and $2 \mathrm{D}$. The prediction works equally well for any $\phi$, the only difference is that the cooling is shifted to earlier/later times depending on the strength of the potential. For 3D, the modification in the cooling rate fits perfectly the cooling of the system as long as it remains homogeneous. For 2D systems there is an appreciable deviation for long times: the theory under-predicts the cooling. The $2 \mathrm{D}$ system is denser $\left(\nu_{2 D}=0.057\right.$ versus $\left.\nu_{3 D}=0.0052\right)$ and that may cause the small difference for large $\tau$ between the theory and the simulations.
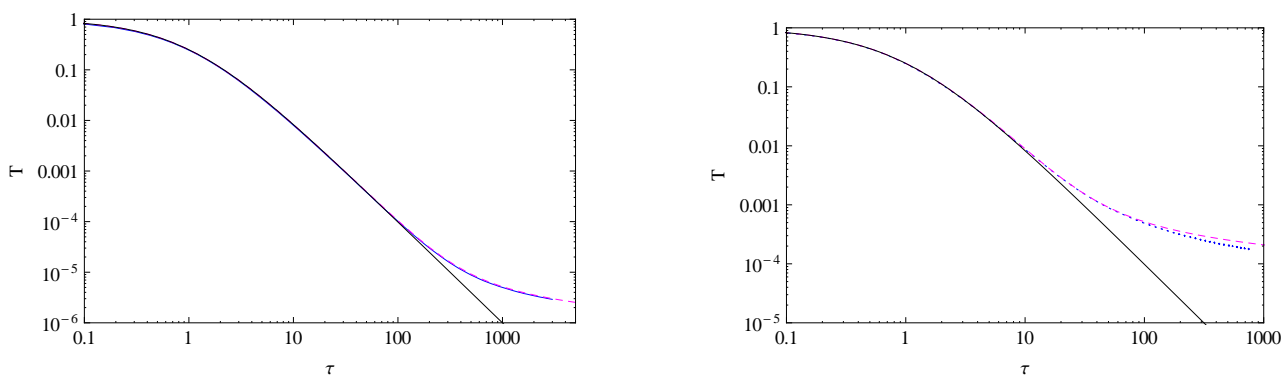

Fig. 11. Cooling in 3D and 2D for the homogeneous case with $\phi=10^{-3}$ and $\phi=10^{-5}$, left and right respectively. The $2 \mathrm{D}$ system is denser $\left(\nu_{2 D}=0.057\right.$ versus $\left.\nu_{3 D}=0.0052\right)$ and that may cause the small difference for large $\tau$ between the theory (magenta dashed line) and the simulations (blue dotted line). The black solid line is Haff's cooling state for reference.

\subsubsection{Non-Homogeneous Cooling}

A more interesting case is when the system is large/dissipative enough to present clusters. If the repulsive potential is weak enough, the time it takes to separate two 
particles that are close to each other is much larger than the time the cluster formation takes to develop, thus allowing for clusters in the system. With other words, if the potential at the beginning is much smaller than the thermal energy, the system has time to develop clusters before the repulsive force separates them. This is similar to the transient cluster formation observed when there is a velocity-dependent coefficient of restitution [35, and will be discussed in subsection 5.2.3.

$$
\Gamma^{-1}=7000
$$

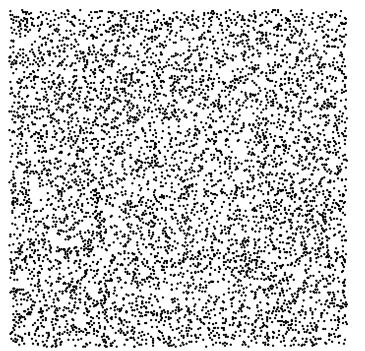

$\Gamma^{-1}=7.3$

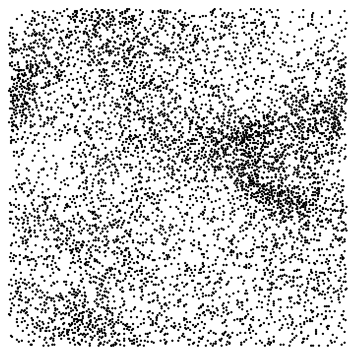

$\Gamma^{-1}=4.15$

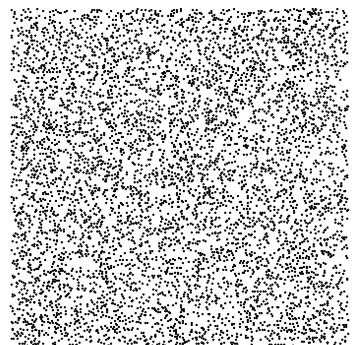

Fig. 12. Simulation snapshots at different $\Gamma$ (time increases from left to right) for a weak repulsive potential $\left(\phi=10^{-4}\right)$ with $N=6400, \nu=0.057$ and $r=0.6$. In the middle picture the transient cluster formation can be seen; in this case, the kurtosis peaks to a high value due to the presence of clusters.

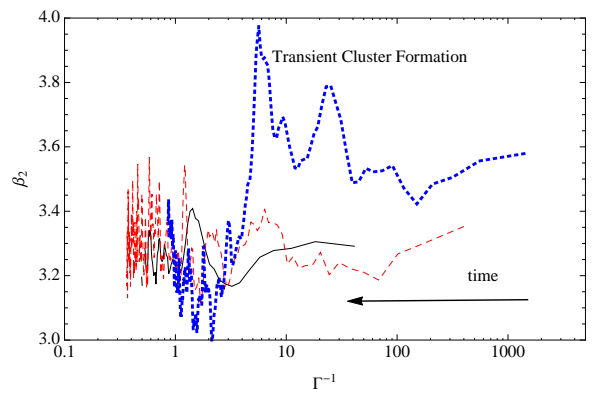

Fig. 13. Kurtosis of the velocity distribution for three systems with $\phi=10^{-3}, 10^{-4}, 10^{-5}$ (solid, dashed and dotted lines) and different coefficients of restitution $r=0.99,0.95$, and 0.9 , respectively, as functions of $\Gamma$. The peak in the kurtosis at $\Gamma^{-1} \simeq 10$ corresponds to the appearance of a transient cluster as the one from Fig. 12, middle (where $r$ is different). Before and after the peak correspond to left and right in the same figure. As $\Gamma$ approaches unity, the velocity distribution tends to decorrelate and $\beta_{2}$ decreases.

Figure 12 shows snapshots of the system at decreasing $\Gamma$ from left to right, for a given repulsive potential and coefficient of restitution. In the middle picture, the transient cluster structures can be seen, while before and after (left and right) the system is homogeneous. This corresponds to the evolution of the system represented in Fig. 13 by the dotted blue line. 
Figure 13 shows the kurtosis of the velocity distribution as a function of $\Gamma$ for three different $\phi$ and coefficient of restitution $r=0.99,0.95,0.9$. If the repulsive potential is weak enough and there is enough dissipation, the cluster instability can develop, making the distribution of velocities much more correlated and hence having a larger kurtosis (dotted line). When the thermal energy is comparable to $\phi$, i.e. $\Gamma \simeq 0.1$, the repulsive force starts to destroy the clusters and produces again a more Maxwellian distribution of the velocities. If the dissipation is small enough, the evolution for different coefficients of restitution in the plane $\Gamma-\beta_{2}$ is similar. The fluctuations around $\Gamma=1$ are due to the long time it takes the system to reach lower temperatures, and hence the evolution appears compressed when plotted as a function of $\Gamma$ instead of $\tau$. The fluctuations look larger than in the repulsive case only because of the different scales of plotting; for large $\Gamma^{-1}$ both oscillate by a similar magnitude.

\subsubsection{Transient cluster formations}

The evolution of the free cooling system with repulsive interactions is somewhat analogous to a system with a velocity dependent coefficient of restitution, where clustering and shearing appear transiently [35. However, the mechanism that controls the presence of clusters is the repulsive force being non-zero and the relative importance of dissipation and repulsion. Any repulsive force between the particles will eventually inhibit cluster formation (whereas attraction and dissipation both work in favour of it). Since there is no external pressure to compress the system, the particles always tend to separate from each other, where stronger dissipation slows down separation. However, this repulsion-related mechanism has a temporal scale controlled by the intensity of the potential at contact, and thus by the available "escape kinetic energy" $T_{e} \propto \phi / \Gamma$. For two spheres touching, the time they need to separate a particle diameter, i.e. to not (mechanically) feel themselves anymore, is $t_{r} \simeq d / \sqrt{2 \phi / m}$. Equivalently, there is a shorter time-scale (for short-range potentials) that involves the range of the potential $t_{\omega} \simeq \omega d / \sqrt{2 \phi / m}$ where $\omega$ is the width of the well in units of particle diameters, which the particles need to leave the range of the potential. Consequently, unless the time of free flight for the particles becomes comparable to or larger than $t_{\omega}$, the repulsive potential is not able to separate the particles and the dynamics is dominated by the hard-sphere properties. Once the collision time-scale of the system exceeds $t_{\omega}$ the clusters begin to disintegrate and the system eventually returns to a homogeneous state.

\subsubsection{Effects of higher density}

For dilute enough systems the cooling rate becomes practically logarithmic, as is the case for a velocity-dependent coefficient of restitution. However, if the density is high enough, the system can reach an elastic regime due to the finite size of the system and the consequent bound for the kinetic energy. For such systems, the evolution is no longer independent of the coefficient of restitution, as it is the case in the dilute regime (data not shown). Since the barrier in the discontinuous case is abrupt, when the temperature is low enough particles cannot overcome the repulsive barrier and stop colliding. Then particles acquire an effective radius equal to the well width and the energy of the system remains constant.

Contrary to the attractive case, increasing the well width would be of no use in trying to recover the physics of the continuous potentials. What is needed in this case is to study how the inclusion of more steps in the potential approaches the continuous case. However, that is beyond the scope of this study. 


\section{Phase diagram for cooling with non-contact interactions}

The phenomenology for the cooling and clustering of hard spheres with short- and long-range non-contact interactions of strength $\phi$ can be summarised as follows: for attractive potentials the freely cooling systems always show clusters (or condensation) on the long term. If $\phi$ is small, relative to the granular temperature, the cooling is initially homogeneous: however, due to the decrease of the granular temperature (cooling), the relative importance of the non-contact interaction increases and eventually leads to clustering. On the other hand, if $\phi$ is large enough, particle pairs stick to each other at first contact already and once pairs are bound together, the cooling continues as a cluster-cluster aggregation process [34]. When a system features a shear- or cluster-instability, it will e.g. show inelastic clusters that cool down slower than the homogeneous hard-sphere case [6 13 15], giving rise to a different dynamics and cluster structure. This slower cooling is not (and cannot be) predicted by the homogeneous mean-field theory that only takes into account the very weak interaction limit case for very low densities.

For the repulsive case, the homogeneous cooling is well predicted by a simple modification of the collision frequency, which implies a modified dissipation rate. If the system is unstable to clustering and the initial $\phi$ is small enough, the system will present inelastic transient clusters, in a way analogous to a granular gas with a velocity-dependent coefficient of restitution. Figure 14 shows a preliminary, qualitative sketch of the phase diagram, for both attractive and repulsive potentials at given density and system size, for different coefficients of restitution and potential strengths.

Qualitative (time-dependent) transition lines can be drawn on the phase diagram by computing the critical coefficient of restitution for the shear instability as a function of $\phi$, for a given system size and density [13. This approach is in analogy to the case of a velocity-dependent coefficient of restitution from Ref. [35], where it was shown that classical stability analysis for the free-cooling gas 3 also holds for a time-dependent dissipation rate. In particular, the eigen-value equation for the shear mode, see Eq. (9) in 35, becomes time-dependent via the dissipation rate. Stability analysis for the critical coefficient of restitution of the shear mode in the dilute limit provides 13: $1-r^{2}=\left(1-r_{c}^{2}\right) / \psi\left(\Gamma^{*}\right)=\left[\alpha / \psi\left(\Gamma^{*}\right)\right] k_{\text {min }}^{2}$, where $r_{c}$ and $\alpha$ represent the classical result, involving a combination of shear-viscosity and dissipation rate pre-factors, valid when $\psi\left(\Gamma^{*}=0\right)=1$, with $\psi$ defined in Eq. (7), and $k_{\min }$ being the smallest wave number possible (the largest wave-length $L_{\max }=2 \pi / k_{\min }$ is the system size). Due to the additional non-contact force, the critical coefficient of restitution will change, since it depends on time via $\psi\left(\Gamma^{*}\right)$, where we have neglected a possible timedependence of $\alpha$. The transition line in the plane $r-\Gamma^{*}$ is then:

$$
\Gamma_{c}^{*}=\Gamma_{c}=\log \left(\frac{1-r^{2}}{1-r_{c}^{2}}\right),
$$

in the repulsive case, for $r<r_{c}$, while it is:

$$
\Gamma_{c}^{*}=-\Gamma_{c}=-\log \left(\frac{1-r^{2}}{1-2 r^{2}+r_{c}^{2}}\right),
$$

in the attractive case, for $r>r_{c}$. Here, $r_{c}$ is the classical result for the critical coefficient of restitution in the given system without non-contact forces.

The phase space can now be represented in two ways: (1) in the $r-\phi$ plane, where our simulations are fixed points, but the transition line sweeps downwards with time, i.e. during the evolution (cooling) of the system; or (2) in the $r-\Gamma^{*}$ plane, 
where the transition lines are invariant, but the systems drift from small to large $\Gamma=\left|\Gamma^{*}\right|$ during cooling.

(1) Figure 14 shows a phase diagram for different systems with fixed $r$ and $\phi$, together with the predicted transition lines $\phi_{c}=\Gamma_{c}^{*} T_{g}(t)$, for different granular temperatures $T_{g}$, sweeping from large to small $|\phi|$ during cooling; the (stable) homogeneous cooling state is predicted to the top and right of the transition lines, as qualitatively well reproduced by the simulations.

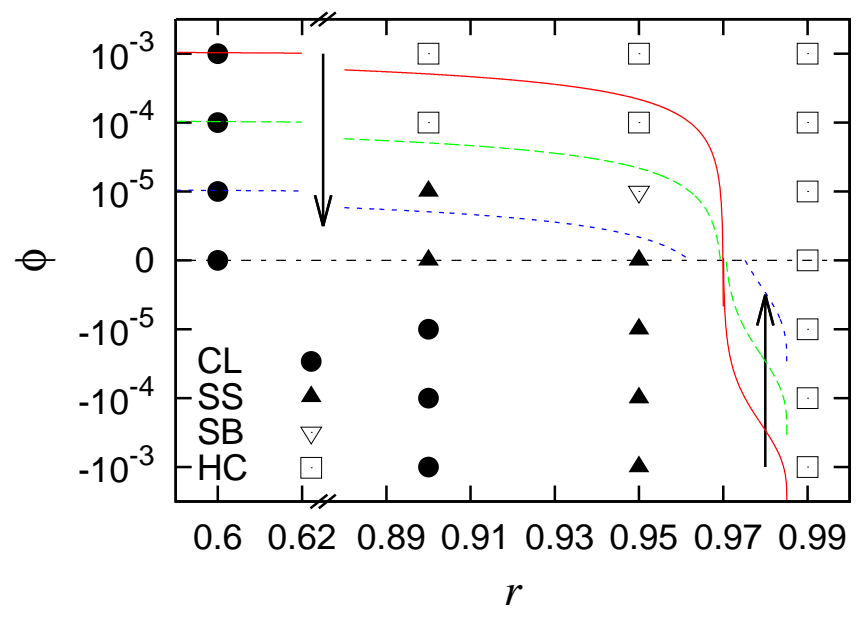

Fig. 14. Phase diagram of free cooling $2 \mathrm{D}$-systems for the potential strengths, $\phi$, where clustering (CL), strong shear (SS), shear banding (SB), and homogeneous cooling (HC) are reported, for different coefficients of restitution, $r$, with $N=6400$ and $\nu=0.057$. For the corresponding hard-sphere systems (points), the classical critical coefficient of restitution is $r_{c}=0.97$, where the upper and lower lines meet. The lines are the transition curves for $T_{g}=10^{-3}$ (red), $10^{-4}$ (green), and $10^{-5}$, (blue). Since the scale is logarithmic, we artificially cut-off at $|\phi|=10^{-6}$ and set the horizontal axis at $\phi=0$; hence not all the curves reach the critical value and thus do not match exactly when crossing from positive to negative $\phi$. The arrows indicate the direction in which the transition lines sweep through the system (towards smaller $|\phi|$ ).

In the repulsive case, a given system can be initially in the stable (top-right) or unstable (bottom-left) zone. Since the transition lines sweep (downwards) towards smaller $\phi$, the former systems will remain stable, whereas the latter will become stable eventually. In the attractive case the unstable domain is larger; a system originally in the stable zone (right, close to $r_{c}$ ) will eventually become unstable, since the transition line sweeps (upwards) towards smaller $|\phi|$, for $\phi<0$.

In summary, this renders repulsive freely cooling systems stable on the long term, whereas attractive systems are expected to become unstable eventually.

(2) Figure 15] shows the $r-\Gamma^{*}$ phase-space where the transition lines are invariant and a freely cooling system moves across them, as indicated by the arrows. A repulsive system, initially in the unstable zone will drift upwards and become eventually stable, whereas an attractive system that could be initially stable will drift downwards and eventually get unstable.

Other, more realistic systems, with a time- or velocity-dependent coefficient of restitution [434435] will also involve an additional side-wards drift of their state 


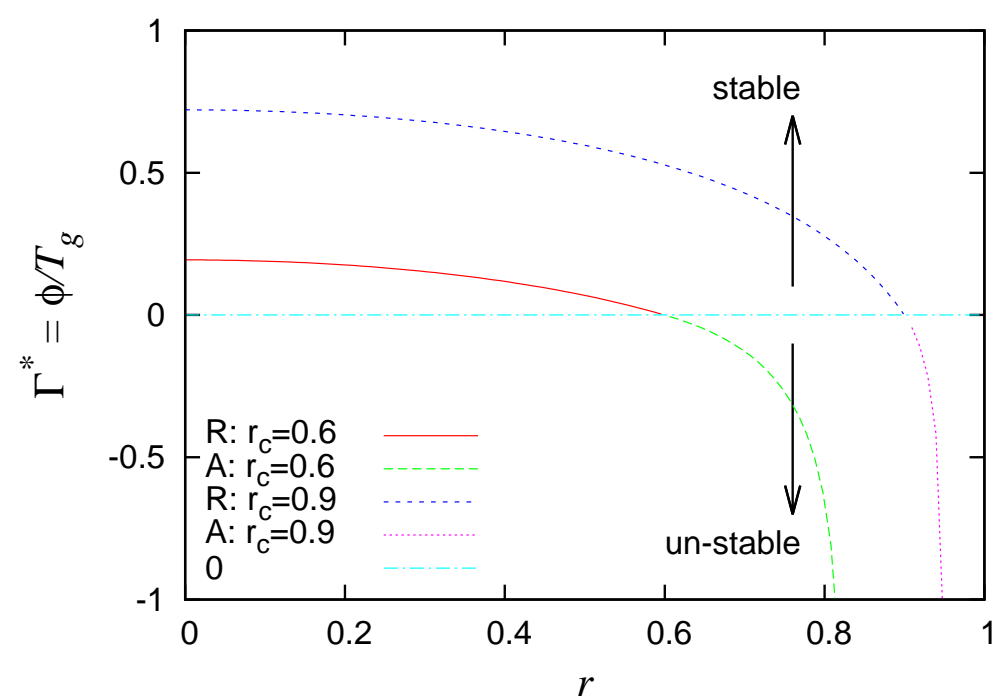

Fig. 15. Phase diagram of free cooling systems for the control parameter $\Gamma^{*}$ and coefficient of restitution, $r$, with $r_{c}=0.9$ and 0.6, from Eqs. (10) and (11).

(data not shown), whereas driven systems in a steady state would be truly stationary in phase space (data not shown).

As final note, each system will require a certain time to react to the hydrodynamic instability of its present state in phase space. When systems drift, there can be a considerable delay time, e.g. when established clusters are expected to be destroyed in the stable region. More realistic systems might display transition lines that are not just functions of $r$ anymore but could allow multiple crossing points; however, this goes beyond the scope of this study.

\section{Conclusions and Outlook}

The free cooling of granular matter with dissipation and - in addition - non-contact attractive or repulsive interactions has been studied by means of event-driven simulations and mean-field hydrodynamic theory. The systems homogeneous cooling behaviour was compared to the modified mean-field theory [20, where the corrected dissipation rate involves the ratio of the interaction potential strength and the granular temperature as the only new control parameter. This theory was developed for 3D; however, we have shown how to apply it for 2D systems. Simulations with discrete potentials confirmed the theoretical predictions for low densities in the repulsive case (in $2 \mathrm{D}$ and $3 \mathrm{D}$ ) but fell short for the attractive case where non-linear effects are more important.

In the present study, in contrast to previous studies 1920, we used different shortranged well- or step-potentials 1338 to complement the simulations with continuous long-range potentials. Remarkably, the theory results that are used here do not have a potential range- or shape-parameter, only the potential magnitude is needed as parameter - at least for small $\Gamma$; since the theory was already tested with the longest range potential $(1 / r)$ one can imagine [20, we consider the present results valid for both short- and long-range potentials. This independence on shape and range was astonishing to the authors and suggests future research towards better theories that do consider shape and/or range of the interactions. 
For attractive potentials the formation of structures, i.e., clusters, is strongly enhanced, as is expected from the results on condensation of elastic hard-spheres with attractive potentials [0 19]; this effect is not predicted by the simple homogeneous mean-field theory. The geometry of the resulting clusters is determined by a complex interplay between dissipation, density, potential strength and range, and the granular temperature intensity that changes during time due to the ongoing cooling.

For repulsive potentials, the theory predicts consistently the cooling behaviour for low dissipation and density as long as the system is homogeneous. This confirms once again that discrete potentials are a good approximation to smooth, continuous interaction potentials in this regime and capture much of the interesting physics of particulate systems. For larger dissipation and weak repulsion we have found that transient clusters appear in the system in a way analogous to the cooling of grains with a velocity dependent coefficient of restitution [434435. This is expected since the dissipation rate in the hydrodynamic equations have a similar time dependence for the free cooling in either case.

Finally, all results point to the great importance of some microscopic parameters in the macroscopic evolution of granular systems and the complex interplay of different micro-mechanic mechanisms that lead to macroscopic, hydrodynamic phenomena. In particular, we showed that the long-time dynamics for a system with both dissipation and non-contact interactions is not simply the superposition of both effects but depends in a more complex way on the particular evolution of the system, its initial state, and the particular parameters that describe it.

\section{Future work:}

The present study started from dissipative systems and added non-contact interactions as new ingredient to predict a phase-diagram that encompasses both mechanisms and their interplay (in the weak interaction limit). A very interesting situation, not considered here, is self-gravity that can lead to clusters without any dissipation, so that dissipation represents a "perturbation", which surely affects the system evolution, as relevant e.g. in astro-physics, and will complement the phase space in the limit $r \rightarrow 1$.

Future work on earth should consider also mixtures of different species of particles with different non-contact interactions as well as more realistic potential-shapes 25]; for example, magnetic (di-polar) interactions [2627. For such potentials and for stronger interactions, relative to the fluctuations, it will be necessary to extended the simple theory used here. The present results are the basis for future research towards a more complete theory for long-range interactions in aerosols or suspensions. Eventually, also driven systems should be considered, see Refs. 2611] and references therein, since they can feature a steady-state in time and should lead to a stable phase-diagram instead of the time-dependent one observed above for cooling granular media.

Eventually, the understanding of the system evolution in its phase space combined with an experimental control of the interaction strength and dissipation (e.g. by changing the surface- or solvent-properties) will allow to better control and optimise engineering processes by controlling at will either the transition lines or the system position in phase space.

\section{Acknowledgments}

We thank H. J. Herrmann for many valuable discussions on dissipative granular systems in the last decades, D. Wolf for additional discussions on systems with noncontact forces, and M. Bannerman for his support concerning the use of DynamO. This study was supported by the Stichting voor Fundamenteel Onderzoek der Materie (FOM), financially supported by the Nederlandse Organisatie voor Wetenschappelijk 
Onderzoek (NWO), through the FOM project 07PGM27, as well by the NWO-STW VICI grant 10828.

\section{References}

1. P. K. Haff. Grain flow as a fluid-mechanical phenomenon. J. Fluid Mech., 134:401-430, 1983.

2. I. Goldhirsch and G. Zanetti. Clustering instability in dissipative gases. Phys. Rev. Lett., 70(11):1619-1622, 1993.

3. S. McNamara. Hydrodynamic modes of a uniform granular medium. Phys. Fluids A, 5(12):3056 - 3070, 1993.

4. S. McNamara and W. R. Young. Dynamics of a freely evolving, two-dimensional granular medium. Phys. Rev. E, 53(5):5089-5100, 1996.

5. H. J. Herrmann, J.-P. Hovi, and S. Luding, editors. Physics of dry granular media NATO ASI Series E 350, Dordrecht, 1998. Kluwer Academic Publishers.

6. S. Luding and H. J. Herrmann. Cluster growth in freely cooling granular media. Chaos, 9(3):673-681, 1999.

7. T. Pöschel and S. Luding, editors. Granular Gases, Berlin, 2001. Springer. Lecture Notes in Physics 564.

8. J. J. Brey, M. J. Ruiz-Montero, and A. Dominguez. Shear state of freely evolving granular gases. Phys. Rev. E, 78(4):041301, 2008.

9. M. N. Bannerman, J. E. Kollmer, A. Sack, M. Heckel, P. Mueller, and T. Pöschel. Movers and shakers: Granular damping in microgravity. Phys. Rev. E, 84:011301, 2011.

10. J. S. Olafsen and J. S. Urbach. Velocity distributions and density fluctuations in a 2D granular gas. Phys. Rev. E, 60:R2468, 1999.

11. S. Luding. Driven granular gases. In T. Pöschel and N. V. Brilliantov, editors, Granular Gas Dynamics, pages 293-316, Berlin, Germany, 2003. Springer.

12. S. McNamara and W. R. Young. Kinetics of a one-dimensional granular medium in the quasielastic limit. Phys. Fluids A, 5(1):34, 1993.

13. S. Miller. Clusterbildung in granularen gasen. PhD-thesis, 2004.

14. S. Miller and S. Luding. Cluster growth in two- and three-dimensional granular gases. Phys. Rev. E, 69:031305, 2004.

15. S. Luding. Structure and cluster formation in granular media. Pramana-Journal of Physics, 64(6):893-902, 2005.

16. N. V. Brilliantov and T. Pöschel. Deviation from maxwell distribution in granular gases with constant restitution coefficient. Phys. Rev. E, 61:2809-2814, 2000.

17. N. Brilliantov and T. Pöschel. Hydrodynamics and transport coefficients for dilute granular gases. Physical Review E, 67:061304, 2003.

18. S. E. Esipov and T. Pöschel. The granular phase diagram. J. Stat. Phys., 86:1385-1395, 1997.

19. M.-K. Müller. Long-range interactions in dilute granular systems. PhD-thesis, 2008.

20. M. K. Müller and S. Luding. Homogeneous cooling with repulsive and attractive longrange potentials. Mathematical Modelling of Natural Phenomena, 6(04):118-150, 2011.

21. A. Brahic. Numerical simulation of a system of colliding bodies in a gravitational field. Journal of Computational Physics, 22(2):171 - 188, 1976.

22. F. G. Bridges, A. Hatzes, and D. N. C. Lin. Structure, stability and evolution of Saturn's rings. Nature, 309:333, 1984.

23. M. Horanyi, G. Morfill, and E. Grun. Mechanism for the acceleration and ejection of dust grains from Jupiter's magnetosphere. Nature, 363(6425):144-146, 1993.

24. T. Pahtz, H. J. Herrmann, and T. Shinbrot. Why do particle clouds generate electric charges? Nat Phys, 6(5):364-368, 2010.

25. S. Dammer and D. E. Wolf. Self-focusing dynamics in monopolarly charged suspensions. Phys. Rev. Lett., 93:150602, 2004.

26. D. L. Blair and A. Kudrolli. Collision statistics of driven granular materials. Phys. Rev. E, 67:041301, 2003. 
27. D. L. Blair and A. Kudrolli. Magnetized granular materials. In H. Hinrichsen and D. E. Wolf, editors, The Physics of Granular Media, pages 281-296, Weinheim, Germany, 2004. Wiley $\mathrm{VCH}$.

28. M. K. Müller and S. Luding. Homogeneous cooling with repulsive and attractive longrange interactions. AIP Conference Proceedings, 1145(1):697-700, 2009.

29. S. Luding. Towards dense, realistic granular media in 2d. Nonlinearity, 22(12):R101R146, 2009.

30. W. F. Carnahan and K. E. Starling. Equation of state for nonattracting rigid spheres. J. Chem. Phys., 51(2):635-636, 1969.

31. D. Henderson. A simple equation of state for hard discs. Molec. Phys., 30(3):971-972, 1975.

32. S. Torquato. Nearest-neighbor statistics for packings of hard spheres and disks. Phys. Rev. E, 51:3170, 1995.

33. V. Ogarko and S. Luding. Equation of state and jamming density for equivalent biand polydisperse, smooth, hard sphere systems. The Journal of Chemical Physics, 136(12):124508, 2012.

34. S. González and S. Luding. Consequences of using different pair-correlation functions on the stability properties of the homogeneous cooling state for a monodisperse system of near-elastic disks. Eur. Phys. J. Special Topics, 179:55-68, 2009.

35. T. Pöschel, N. V. Brilliantov, and T. Schwager. Transient clusters in granular gases. J. Phys.-Cond. Matter, 17(24):S2705-S2713, 2005.

36. T. Scheffler and D. E. Wolf. Collision rates in charged granular gases. Granular Matter, 4(3):103-113, 2002.

37. S. González, A. R. Thornton, and S. Luding. An event-driven algorithm for fractal cluster formation. Computer Physics Communications, 182(9):1842 - 1845, 2011.

38. M. N. Bannerman, R. Sargant, and L. Lue. DynamO: A free $\mathcal{O}(N)$ general event-driven molecular dynamics simulator. Journal of Computational Chemistry, 32(15):3329-3338, 2011.

39. P. Meakin and Z. R. Wasserman. Some universality properties associated with the cluster-cluster aggregation model. Physics Letters A, 103(67):337 - 341, 1984.

40. N. G. van Kampen. Condensation of a classical gas with long-range attraction. Phys. Rev., 135(2A):A362-A369, 1964.

41. V. Y. Zaburdaev, M. Brinkmann, and S. Herminghaus. Free cooling of the onedimensional wet granular gas. Phys. Rev. Lett., 97:018001, 2006.

42. S. Ulrich, T. Aspelmeier, K. Roeller, A. Fingerle, S. Herminghaus, and A. Zippelius. Cooling and aggregation in wet granulates. Phys. Rev. Lett., 102:148002, 2009.

43. S. McNamara and S. Luding. Energy nonequipartition in systems of inelastic, rough spheres. Phys. Rev. E, 58:2247-2250, 1998.

44. S. Luding and A. Goldshtein. Collisional cooling with multi-particle interactions. Granular Matter, 5(3):159-163, 2003. 